\title{
Multi-Objective Optimization Design of Radiation Shadow Shield for Space Nuclear Power With Genetic Algorithm
}

\author{
Bin $\mathrm{Liu}^{1 *}$, Huanwen $L v^{1}$, Lan $\mathrm{Li}^{1}$, Xiaoming Chai ${ }^{1 *}, \mathrm{Hu} X \mathrm{Xu}^{2 *}, \mathrm{Yi} \operatorname{Tan}^{1}$, Bangyang Xia ${ }^{1 *}$ and \\ Cong Chen ${ }^{1}$
}

${ }^{1}$ Science and Technology on Reactor System Design Technology Laboratory, Nuclear Power Institute of China, Chengdu, China, ${ }^{2}$ School of Nuclear Science and Technology, Xi'an Jiaotong University, Xi'an, China

OPEN ACCESS

Edited by: Mingfei Yan,

Riken, Japan

Reviewed by:

Zhangpeng Guo, North China Electric Power University,

China

Pan Wu,

Xi'an Jiaotong University, China

Zhihua Liu,

University of Illinois at UrbanaChampaign, United States

*Correspondence:

Bin Liu

liubin871204@126.com

Xiaoming Chai

chaixm@163.com

$\mathrm{Hu} \times$

xuhu_xu@126.com

Bangyang Xia

xiabangyang@163.com

Specialty section: This article was submitted to Nuclear Energy,

a section of the journal Frontiers in Energy Research.

Received: 24 October 2021 Accepted: 10 January 2022 Published: 17 February 2022

Citation:

Liu B, Lv H, Li L, Chai X, Xu H, Tan Y, $X i a B$ and Chen $C$ (2022) MultiObjective Optimization Design of

Radiation Shadow Shield for Space Nuclear Power With Genetic Algorithm.

Front. Energy Res. 10:800930. doi: 10.3389/fenrg.2022.800930
The radiation shadow shield of the space nuclear power is an important component to protect the electronic device at the dose plane. In this paper, design of the radiation shadow shield is first modeled as a multi-objective optimization problem, and then converted into a single objective optimization problem by setting the other sub-objectives as constraints. An optimization method combining a single objective genetic algorithm with Monte Carlo simulation is developed to solve the optimization problem of shadow shield design. Through optimization, weight of the radiation shadow shield can be greatly reduced $(14.7 \%$ of the weight of the initial design and $16.4 \%$ of the weight of the best individual of the first generation), while radiation dose at the dose plane is lower than the acceptable tolerance. The optimization method developed in this work is an automated optimization strategy by searching for the parameter space, which will not be subject to human preferences. The global optimal solutions can be obtained without the tedium of human manual work.

Keywords: space nuclear power, fission reactor, radiation shadow shield, multi-objective optimization, genetic algorithm

\section{INTRODUCTION}

Deep space exploration and immense developments in space science and technology demand reliable, long life, and high-power energy sources. The fission nuclear reactor, due to its highpower density, compact configuration, and long lifetime deployment, is a suitable choice for the harsh environments of the outer planets. Many projects for space reactors ranging from low to high power have been constructed, such as the BUK and TOPAZ series (Stanculescu, 2005; ElGenk, 2009; Grasty, 1978; Johnson, 1982; Bennett, 1989; Sidiqi, 1999), the SNAP series, and the SP100 reactor (Angelo and Buden, 1985; Demuth, 2003; Bruno, 2008). A typical schematic of the space nuclear reactor is shown in Figure 1. The shadow shield is designed to protect the electronic system of the spacecraft from neutron and gamma radiation generated by the reactor core. Hydrogen contained materials such as $\mathrm{LiH}$ and $\mathrm{ZrH}_{2}$ are usually chosen to slow down neutrons and heavy materials such as steel, lead, and tungsten are chosen to attenuate gamma rays. In recent years, tungsten doping with boron cardide $\left(\mathrm{W}-\mathrm{B}_{4} \mathrm{C}\right)$ is used in shadow shield of space nuclear reactor, to attenuate gamma rays as well as to absorb neutrons (Ahmad et al., 2021). The radiation shadow shield is a considerable part of the total mass of the space nuclear reactor. The mass of the shadow shield in the designs of Kilopower, Topaz II, and SNAP-10A is $42.6 \%, 36.8 \%$, and $17.4 \%$ of the mass of the whole nuclear system, respectively (Voss, 1984; Gibson et al., 2015). In addition, the total mass of the space nuclear reactor is closely related to the launching cost. 


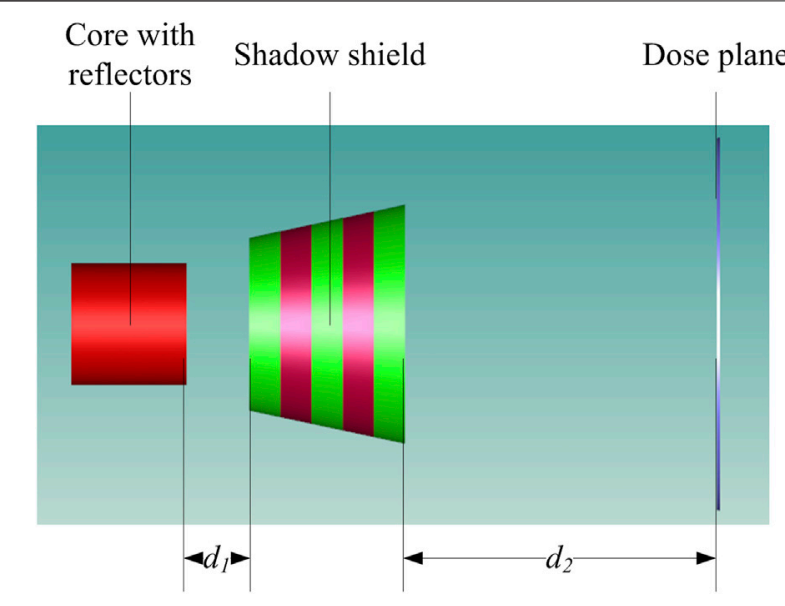

FIGURE 1 | Schematic of the shadow shield of the space nuclear reactor.

$D_{1}$ is the distance between the reactor and the shield to provide space for heat pipes to bend outside of the shield; $d_{2}$ is the distance between the shield and the dose plane, where electronic systems are placed.

Currently, design of the shadow shield is mainly subject to the designer's expertise, which is essentially a manual iterative procedure using both the deterministic and Monte Carlo codes (Ahmad et al., 2021; Woodrow, 1987). The current design method is timeconsuming, low-efficiency, and the optimal solution is always hard to obtain. Genetic algorithm is a stochastic method with the ability to find true or approximate solutions to the global optimization. In the recent years, genetic algorithm is applied to nuclear-related applications, such as the nuclear fuel loading and reloading optimization (Sobolev et al., 2017) and shielding design of the PWRs (pressurized water reactors) (Chen et al., 2019; Chen et al., 2020). In this paper, the typical multi-objective optimization problem of the shadow shield design of the space nuclear reactor is converted into a single-objective optimization problem by setting the rest of the sub-objectives as constraints. In addition, the optimization method combining genetic algorithm with the MCNP code is developed to solve the optimization problem of the shadow shield design.

\section{METHODOLOGY}

\section{The Optimization Problem of the Shadow Shield Design}

The purpose of the shadow shield of the space nuclear reactor is to protect the electronic device placed at the dose plane from radiation produced in the reactor core. Moreover, the shadow shield needs to be as light and compact as possible to reduce the launching cost. As a result, the target of the shadow shield design is to obtain a set of parameters, such as geometrical parameters of the shadow shield, making the weight of the shadow shield, energy deposition of gamma rays in silicon, and fast neutron fluence equivalent in silicon at the dose plane minimal, which can be described mathematically as

$$
\begin{array}{r}
\min f(\vec{x})=\left(f_{W}(\vec{x}), f_{g}(\vec{x}), f_{n}(\vec{x})\right)^{T} \\
\text { s.t. }\left\{\begin{array}{l}
\vec{x}=\left(x_{1}, x_{2}, \cdots, x_{n}\right), \vec{x} \in \mathbb{R}^{n} \\
L_{j} \leq x_{j} \leq U_{j}(j=1,2, \cdots, n) \\
h_{k}\left(x_{j}\right) \leq 0(k=1,2, \ldots, q)
\end{array}\right.
\end{array}
$$

where $f_{W}(\vec{x}), f_{n}(\vec{x})$, and $f_{g}(\vec{x})$ represent weight of the shadow shield, fast neutron fluence equivalent in silicon and energy deposition of gamma rays in silicon, respectively; $\vec{x}$ is an $n$-dimensional variable vector of the design parameters; $L_{j}$ and $U_{j}$ represent the lower and upper bounds of the variables; $h_{k}$ represents the inequality constraints of the design parameters.

Equation 1 is the typical multi-objective optimization problem. As to shadow shield design of space reactors, the purpose is to obtain the optimal parameters with the minimum weight while the neutron and gamma radiation are at the dose plane lower than the tolerable limits, so the multiobjective optimization problem stated by Equation 1 can be converted into a single-objective optimization problem, given as:

$$
\begin{gathered}
\min f_{W}(\vec{x}) \\
\text { s.t. }\left\{\begin{array}{l}
f_{g}(\vec{x}) \leq f_{g, \text { limit }}(\vec{x}) \\
f_{n}(\vec{x}) \leq f_{n, \text { limit }}(\vec{x}) \\
\vec{x}=\left(x_{1}, x_{2}, \cdots, x_{n}\right), \vec{x} \in \mathbb{R}^{n} \\
L_{j} \leq x_{j} \leq U_{j}(j=1,2, \cdots, n) \\
h_{k}\left(x_{j}\right) \leq 0(k=1,2, \ldots, q)
\end{array}\right.
\end{gathered}
$$

where $f_{g \text {,limit }}$ and $f_{n \text {,limit }}$ are the tolerable limits of the gamma dose and the neutron fluence in silicon.

Equation 2 can be converted into the maximization problem by modifying the objective function, given as,

$$
\max g_{W}(\vec{x})=\frac{1}{1+f_{W}(\vec{x})}
$$

\section{The Evolution Operators of Genetic Algorithm}

Genetic algorithm is employed to solve the single objective optimization problem given by Equation 3 for its ability to obtain the global optimization solution (Holland, 1992). The population evolves via the evolution operators through many generations, breeding strong solutions while weeding out weak solutions to find the global optimal solution. The evolution operators involve encoding and decoding of the candidate solutions, fitness evaluation, parent selection, mating, and mutation (De Jong, 1975; Goldberg, 1989). Binary encoding method is adopted for its simplicity for implementation and designing the genetic operators (Zhou and Sun, 1999). The optimization parameter $x$ is expressed with the binary strings $a_{l} a_{l-1} \cdots a_{1}$. The corresponding decoding formula is given as

$$
x=x_{\min }+\left(\sum_{i=1}^{l} a_{i} \cdot 2^{i-1}\right) \cdot \frac{x_{\max }-x_{\min }}{2^{l}-1}
$$

The genetic algorithm search operated on the Darwinian principle of "survival of the fittest". Hence fitness is a measure of how well an 


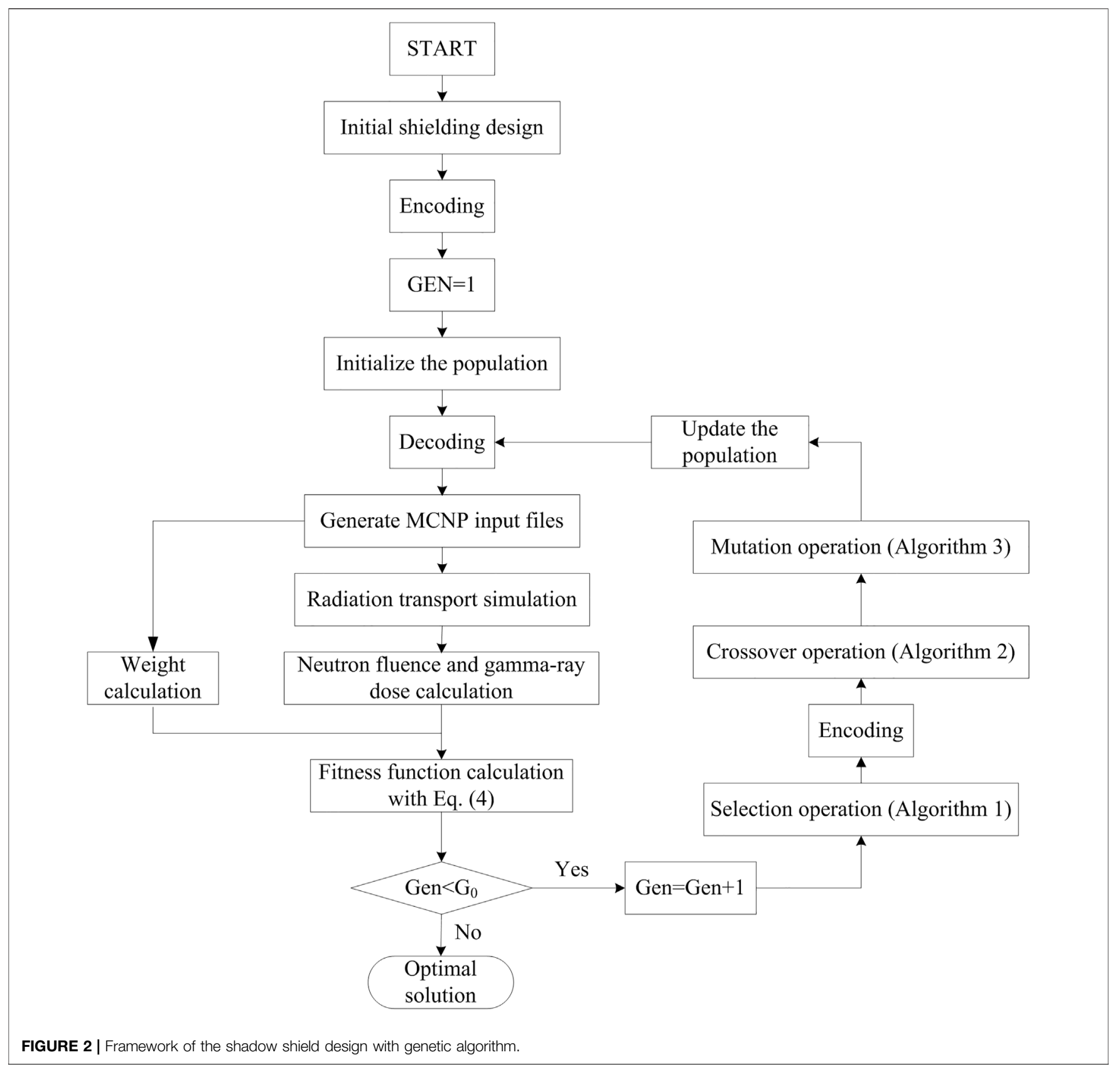

individual performs in its environment. In order to implement constraints of the sub-objectives during the optimization, candidates not satisfying the constraints are treated as lethal genes (Zhou and Sun, 1999), of which fitness values are set to be zeros, given as,

$$
f i t(\vec{x})=\left\{\begin{array}{l}
g_{W}(\vec{x}), \text { if } f_{g}(\vec{x}) \leq f_{g, \text { limit }}(\vec{x}) \text { and } f_{n}(\vec{x}) \leq f_{n, \text { limit }}(\vec{x}) \\
0, \quad \text { else }
\end{array}\right.
$$

Equation 5 ensures candidates not satisfying constraints are eliminated in the next generations, and eventually converts the multi-objective optimization problem of shadow shield design of space nuclear reactor into a single-objective optimization problem.

Parent selection is random but biased such that the probability of selecting a candidate solution to be a parent increases with increasing fitness. In this work, parents are selected using a technique known as tournament selection. With this technique, "tournaments" are held between randomly selected pairs of solutions; the solution with the highest fitness wins the tournament and is selected to be a parent. The process is repeated to find the mate (Zhou and Sun, 1999; Luke, 2016). Moreover, parents mate by interchanging parts of their encoded chromosome structure using a technique known as "crossover". The crossover technique involves randomly selecting a site along the chromosome string as a crossover point. All the genetic information following that point is exchanged with the mate. The "two-point crossover" technique is employed in this work (Luke, 2016). The offspring solutions next experience mutation. Mutation is carried out by randomly and infrequently changing the state of one or 
more bits within a chromosome. Sometimes the mutation produces a favorable effect by increasing performance, sometimes not. Bit-Flip mutation method is employed in this paper (Luke, 2016).

\section{Framework of the Shadow Shield Design Method}

The optimization process of the shadow shield design is illustrated in Figure 2. The Monte Carlo code MCNP is applied to radiation transportation simulation for its ability to model the complexity geometry (X-5 Monte Carlo team, 2003). Moreover, the conversion factors provided by energy response function (DE/DF) cards allow MCNP to convert the predicted particle fluences to neutron fluence equivalent in silicon (DePriest, 2014). The framework of the shadow shield optimization design using genetic algorithm is depicted in Figure 2. First, an initial solution is given to start the optimization according to the initial structural and material parameters; then, a certain number of shield solutions are randomly generated to form an initial population. Generate MCNP input files for shield solutions in the population and calculate the shadow shield weight according to the corresponding parameters, and then perform the radiation transport simulations with the MCNP code to calculate neutron fluence and gamma-ray dose. Then the fitness functions are calculated with Equation 5 and the population evolutions are performed employing the selection, crossover, and mutation operators illustrated in The Evolution Operators of Genetic Algorithm to update the MCNP input files until reaching the maximum generation.

MCNP simulations are performed with a multi-group and parallel computing mode. Besides, the based variance reduction technique utilized in MCNP runs to obtain satisfactory results with acceptable errors less than $10 \%$. As a result, the MCNP simulations in the optimization procedure will obtain satisfactory results within an acceptable computational time.

\section{OPTIMIZATION RESULTS}

\section{Reactor Model}

The reactor is a typical fast reactor of $100 \mathrm{~kW}$ th power. The equivalent diameter and height of the reactor are $18 \mathrm{~cm}$ and $16 \mathrm{~cm}$, respectively. The core consists of enriched UN fuel ( $90 \%$ enriched $\left.{ }^{235} \mathrm{U}\right)$, heat pipes
TABLE 1 | The composition of the shielding materials (Wt\%).

\begin{tabular}{|c|c|c|}
\hline & $\mathrm{LiH}\left(0.8 \mathrm{~g} / \mathrm{cm}^{3}\right)$ & $W-B_{4} C\left(11.59 \mathrm{~g} / \mathrm{cm}^{3}\right)$ \\
\hline${ }^{6} \mathrm{Li}$ & $5.72 \mathrm{E}-02$ & - \\
\hline${ }^{7} \mathrm{Li}$ & 8.17E-02 & - \\
\hline $\mathrm{H}$ & 1.26E-01 & - \\
\hline${ }^{10} \mathrm{~B}$ & - & 7.04E-02 \\
\hline${ }^{11} \mathrm{~B}$ & - & 7.83E-03 \\
\hline $\mathrm{C}$ & - & 2.17E-02 \\
\hline${ }^{182} \mathrm{~W}$ & - & 2.36E-01 \\
\hline${ }^{183} \mathrm{~W}$ & - & $1.29 \mathrm{E}-01$ \\
\hline${ }^{184} \mathrm{~W}$ & - & 2.76E-01 \\
\hline${ }^{186} \mathrm{~W}$ & - & 2.59E-01 \\
\hline
\end{tabular}

containing the liquid lithium to extract heat and control rods of $\mathrm{B}_{4} \mathrm{C}$ to control the reactivity. The $\mathrm{BeO}$ is chosen as a reflector with a thickness of $10 \mathrm{~cm}$. The shadow shield is in the shape of a truncated cone that makes the half-angle of $12^{\circ}$ on the face of the front reflector and shadows the electronic devices from radiation. The distance between the reactor and the shadow shield $\left(d_{1}\right.$ in Figure 1$)$ is $20 \mathrm{~cm}$ to provide space for heat pipes to bend outside of the shield, and the distance between the shield and the electronic system $\left(d_{2}\right.$ in Figure 1$)$ is $100 \mathrm{~cm}$.

\section{The Optimization Parameters}

The lithium hydride $(\mathrm{LiH})$ is chosen to slow down neutrons, and the composite material $\mathrm{W}-\mathrm{B}_{4} \mathrm{C}\left({ }^{10} \mathrm{~B}\right.$ enriched) is chosen to attenuate gamma rays as well as absorb neutrons. The compositions of $\mathrm{LiH}$ and $\mathrm{W}-\mathrm{B}_{4} \mathrm{C}$ are listed in Table 1 . The enrichment of ${ }^{10} \mathrm{~B}$ is $90 \%$.

The axial and radial profiles of the shadow shield are shown in Figure 3. The structure of the shadow shield is designed as " $\mathrm{LiH}$, $\mathrm{W}-\mathrm{B}_{4} \mathrm{C}, \mathrm{LiH}, \mathrm{W}-\mathrm{B}_{4} \mathrm{C}, \mathrm{LiH}^{\prime}$. The thickness of each layer is treated as the optimization parameters of the genetic algorithm, such as $t_{1}, t_{2}, t_{3}, t_{4}, t_{5}$, shown in Figure 3A. Six control apertures are designed within the shadow shield for the control rods system, shown in Figure 3B.

\section{The Shielding Design Limits}

The target of the shadow shield is to protect the electronic systems from radiation from the reactor core. According to the study in the El-Genk's literature, the tolerable limits of neutron fluence and gamma dose for the silicon are $1.0 \times 10^{12} \mathrm{nvt}$ (fast neutrons $>0.1 \mathrm{MeV}$ ) and 1.0 Mrad, respectively (Ahmad et al., 2021; El-

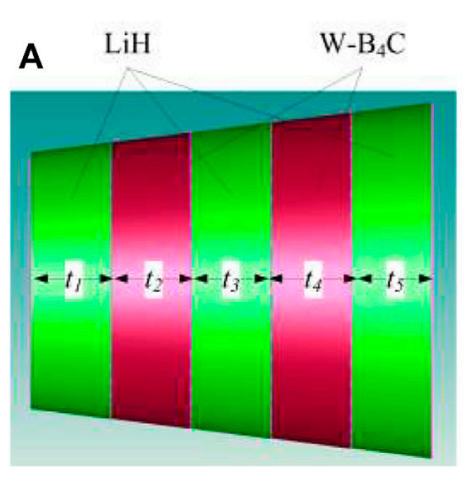

Control aperture

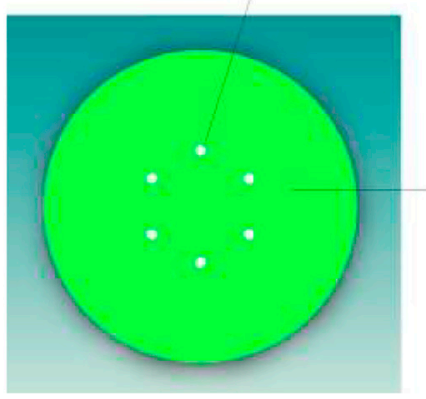

Shielding

material

FIGURE 3 | The axial (A) and the radial (B) profiles of the shadow shield. 


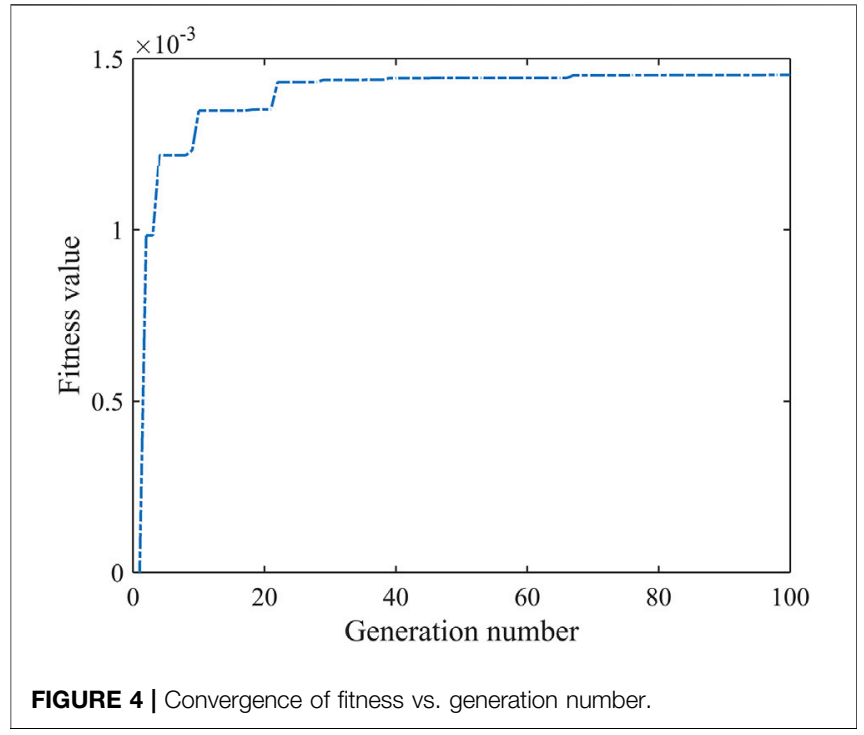

Genk et al., 2005). Considering the 10 years as design operational lifetime of the reactor, the limits for fast neutron flux and gamma dose rate at dose plane are $3.17 \times 10^{3} \mathrm{n} \cdot \mathrm{cm}^{-2} \cdot \mathrm{s}^{-1}$ and $3.17 \times$ $10^{-3} \mathrm{rad}^{-1}$, respectively.

\section{The Optimization Results}

The genetic algorithm performs 100 generations with the population size of 50 . The crossover rate and the mutation rate of the genetic algorithm are 0.7 and 0.5 , respectively. Convergence of fitness value vs. generation number of the optimization is shown in Figure 4. The fitness value grows slowly while the generation reaches 70 , so 100 generations are sufficient to the optimization.
Ranges of the optimization parameters are $0-150 \mathrm{~cm}$. For compact design, the constraint of the optimization parameters, i.e., $h\left(x_{j}\right)$ in Equation 1 is written as:

$$
\sum_{i=1}^{n=5} t_{i}-150 \leq 0
$$

The optimization method starts with the initial shielding design, and then a certain number of shield solutions are randomly generated to form an initial population, and through the genetic operators with many generations, the optimal individual is obtained. As a result, the objectives and parameters of three cases are studied, such as the initial design (Case 1), the best individual of the first generation (Case 2), and the optimal solution (Case 3). The optimization objectives and the parameters of the three cases are listed in Table 2, where $f_{n}(\vec{x})$ and $f_{g}(\vec{x})$ are calculated with the MCNP code with relative errors listed within the bracket below the objective values.

The sub-objective $f_{W}(\vec{x})$ is competing with the sub-objectives $f_{n}(\vec{x})$ and $f_{g}(\vec{x})$. As is shown in Table 2, the sub-objectives $f_{n}(\vec{x})$ and $f_{g}(\vec{x})$ of Case 1 and Case 2 are much less than the tolerable limits, indicating that the objective $f_{W}(\vec{x})$ can be future optimized. However, sub-objectives $f_{n}(\vec{x})$ and $f_{g}(\vec{x})$ of Case 3 are slightly lower than the tolerable limits, indicating that $f_{W}(\vec{x})$ of Case 3 is near the global optimal solution. The structures of the three cases are shown in Figure 5. The structure of case 1 is shown in Figure 5A, and the structure of case 2 is shown in Figure $5 \mathbf{B}$. The optimal structure is "LiH, W-B4C, $\mathrm{LiH}$," shown in Figure 5C. Both light material $(\mathrm{LiH})$ and heavy material $(\mathrm{W}-\mathrm{B} 4 \mathrm{C})$ are needed to shield the neutron and gamma mixing field of the nuclear reactor. Among the combinations of $\mathrm{LiH}$ and $\mathrm{W}-\mathrm{B} 4 \mathrm{C}$, the optimized structure is the most likely structure to obtain the minimal weight to ensure the radiation at the dose plane is below the tolerable limits. Compared

TABLE 2 | Objectives and parameters of the initial design, best individual of the first generation and the optimized solution.

\begin{tabular}{|c|c|c|c|c|c|c|c|c|}
\hline & $\begin{array}{c}\mathbf{f}_{\mathrm{w}}(\overrightarrow{\mathbf{x}}) \\
(\mathbf{k g})\end{array}$ & $\begin{array}{c}f_{n}(\vec{x}) \\
\left(n / \mathrm{cm}^{2} / s\right)\end{array}$ & $\begin{array}{c}\mathbf{f}_{\mathbf{g}}(\overrightarrow{\mathbf{x}}) \\
(\mathrm{rad} / \mathrm{s})\end{array}$ & $\begin{array}{c}t_{1} \\
(\mathrm{~cm})\end{array}$ & $\begin{array}{c}t_{2} \\
(\mathrm{~cm})\end{array}$ & $\begin{array}{c}t_{3} \\
(\mathrm{~cm})\end{array}$ & $\begin{array}{c}t_{4} \\
(\mathrm{~cm})\end{array}$ & $\begin{array}{c}t_{5} \\
(\mathrm{~cm})\end{array}$ \\
\hline Range & - & - & - & $0-150$ & $0-150$ & $0-150$ & $0-150$ & $0-150$ \\
\hline Case 1 & 4722.5 & $1.22 E+05(0.04)$ & 3.54E-05 (0.04) & 30 & 30 & 30 & 30 & 30 \\
\hline Case 2 & 4208.7 & $1.75 E+05(0.03)$ & 5.02E-05 (0.04) & 13.3 & 44.6 & 15.4 & 25.0 & 23.5 \\
\hline Case 3 & 691.9 & $3.06 E+05(0.03)$ & $0.0031(0.01)$ & 30.0 & 9.5 & 18.6 & 0.0 & 26.0 \\
\hline
\end{tabular}

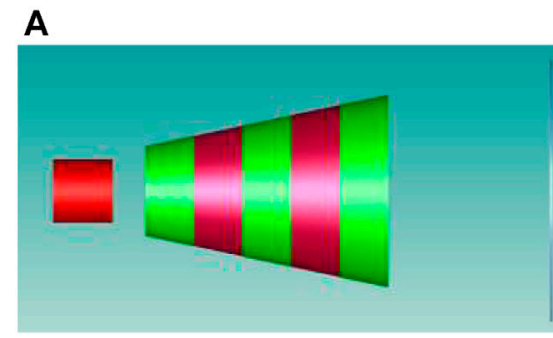

case 1

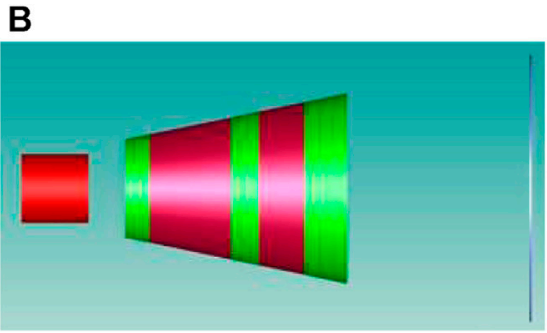

case 2
C

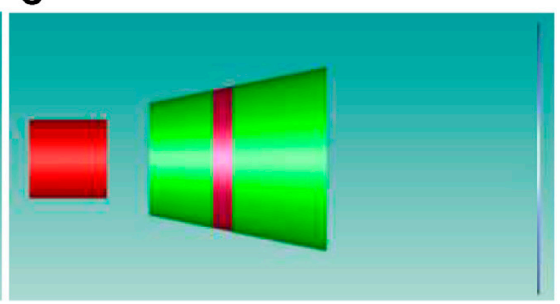

case 3

FIGURE 5 | The structures of Case 1 (A), Case 2 (B), and Case 3 (C). 
with Case 1 and Case 2, the weight of the shadow shield is much less when the radiation at the dose plane is below the tolerable limits.

\section{CONCLUSION}

The radiation shadow shield of the space nuclear reactor is a vital component to protect electronic devices at the dose plane from radiation from the reactor core. The radiation shadow shield needs to be lightweight and compact to reduce the launching cost. The multi-objective optimization problem of designing the shadow shield is converted into a single-objective optimization problem by setting the sub-objectives as constraints. An optimization method combining a single objective genetic algorithm with Monte Carlo simulation is developed to solve the radiation shadow shield designing problem. Compared with the initial design and the best individual of the first generation, weight of the optimal solution is greatly reduced $(14.7 \%$ of the weight of the initial design and $16.4 \%$ of the weight of the best individual of the first generation). In addition, the radiation dose at the dose plane of the optimal solution is lower than the acceptable tolerance. The optimization method developed in this work is an automated optimization strategy by searching

\section{REFERENCES}

Ahmad, S., Chang, B., Li, B., Yang, Q., and Liu, C. (2021). Mass Optimization of the Radiation Shadow Shield for Space Nuclear Power System. Prog. Nucl. Energ. 131, 103607-1036078. doi:10.1016/j.pnucene.2020.103607

AngeloJr., and Buden, D. (1985). Space Nuclear Power. United States.

Bennett, G. L. (1989). Soviet Space Nuclear Reactor Incidents: Perception versus Reality. Space Nucl. Power Syst. 10, 273. doi:10.2514/6.2019-4030

Bruno, C. (2008). Nuclear Space Power and Propulsion Systems. Prog. Astronautics Aeronautics 26, 116-118. doi:10.2514/4.860096

Chen, Z., Zhang, Z., Xie, J., Guo, Q., and Yu, T. (2019). Metaheuristic Optimization Method for Compact Reactor Radiation Shielding Design Based on Genetic Algorithm. Ann. Nucl. Energ. 134, 318-329. doi:10.1016/j.anucene.2019.06.031

Chen, Z., Zhang, Z., Xie, J., Guo, Q., Yu, T., Zhao, P., et al. (2021). Multi-objective Optimization Strategies for Radiation Shielding Design with Genetic Algorithm. Comp. Phys. Commun. 260, 107267-1076275. doi:10.1016/j.cpc.2020.107267

De Jong, K. A. (1975). An Analysis of the Behavior of a Class of Genetic Adaptive Systems. Ph. D. dissertation. Ann Arbor: University of Michigan.

Demuth, S. F. (2003). SP100 Space Reactor Design. Prog. Nucl. Energ. 42 (3), 323-359. doi:10.1016/S0149-1970(03)90003-5

DePriest, K., R. (2014). Impact of ASTM Standard E722 Update on Radiation Damage Metrics. Albuquerque, NM: SANDIA Report, SAND2014-5005.

El-Genk, M., Hatton, S., Fox, C., and Tournier, J. M. (2005). Jsme International Journal. Ser. B. Fluids \& Thermal Engineering. Albuquerque, NM: AIP Publishing, 4730484. doi:10.1063/1.1867163SCoRe - Concepts of Liquid Metal Cooled Space Reactors for Avoidance of Single-Point Failure

El-Genk, M. S. (2009). Deployment History and Design Considerations for Space Reactor Power Systems. Acta Astronautica 64 (9-10), 833-849. doi:10.1016/j. actaastro.2008.12.016

Gibson, M. L. S., Mason, C. L., Bowman, D. I., Postan, P. R., McClure, J. Creasy., and Robinson, C. (2015). "Development of NASA's Small Power System for Science and Human Exploration," in 12th International Energy Conversion Engineering Conference.

Goldberg, D. (1989). Genetic Algorithms in Search, Optimization and Machine Learning. Addison-Wesley Pub. Co.

Grasty, R. L. (1978). Estimating the Fallout on Great Slave lake from COSMOS954. Trans. Am. Nucl. Soc. 30. doi:10.1016/B978-1-85617-752-8.10026-1

Holland, J. H. (1992). Adaptation in Nature and Artificial Systems. MIT Press. for the parameter space, which will not be subject to human preferences. The global optimal solutions can be obtained without the tedium of human manual work.

\section{DATA AVAILABILITY STATEMENT}

The raw data supporting the conclusion of this article will be made available by the authors, without undue reservation.

\section{AUTHOR CONTRIBUTIONS}

BL designed the research and wrote the paper; HL conducted the research; HX performed the Monte Carlo simulations; XC and LL raised valuable questions that help improving the paper; BX, YT, and CC provided good suggestions for data analysis.

\section{FUNDING}

This research was supported by National Natural Science Foundation of China under Grant No. 11905213.

Johnson, N. L. (1982). Orbital Phasings of Soviet Ocean Surveillance Satellites. J. Spacecraft Rockets 19 (2), 113-117. doi:10.2514/3.62218

Lones, M. (2011). Sean Luke: Essentials of Metaheuristics. Genet. Program Evolvable Mach 12 (3), 333-334. doi:10.1007/s10710-011-9139-0

Sidiqi, A. (1999). Staring at the Sea-The Soviet RORSAT and EORSAT Programmes. J. Br. Interplanetary Soc. 52, 397-416. doi:10.1007/978-1-4419-6938-5_9

Sobolev, A. V., Gazetdinov, A. S., and Samokhin, D. S. (2017). Genetic Algorithms for Nuclear Reactor Fuel Load and Reload Optimization Problems. Nucl. Energ. Tech. 3 (2), 231-235. doi:10.1016/j.nucet.2017.07.002

Stanculescu, A. (2005). The Role of Nuclear Power and Nuclear Propulsion in the Peaceful Exploration of Space. Vienna: IAEA.

Voss, S. S. (19841983). Air Force Weapons Lab. NM: Kirtland AFB.Snap Reactor Overview Woodrow, L. L. (1987). Shielding Analysis of a Small Compact Space Nuclear Reactor. Kirtland AFB, NM: Air force weapon laboratory.

X-5 Monte Carlo Team (2003). MCNP-A General N-Particle Transport Code. Santa Fe,NM: Los Alamos National Laboratory.

Zhou, M., and Sun, S. D. (1999). Genetic Algorithms: Theory and Applications. Beijing: National Industrial Press.

Conflict of Interest: The authors declare that the research was conducted in the absence of any commercial or financial relationships that could be construed as a potential conflict of interest.

The reviewer PW declared a shared affiliation with one of the authors, HX, to the handling editor at time of review.

Publisher's Note: All claims expressed in this article are solely those of the authors and do not necessarily represent those of their affiliated organizations, or those of the publisher, the editors, and the reviewers. Any product that may be evaluated in this article, or claim that may be made by its manufacturer, is not guaranteed or endorsed by the publisher.

Copyright (c) $2022 \mathrm{Liu}, \mathrm{Lv}, \mathrm{Li}$, Chai, Xu, Tan, Xia and Chen. This is an open-access article distributed under the terms of the Creative Commons Attribution License (CC BY). The use, distribution or reproduction in other forums is permitted, provided the original author(s) and the copyright owner(s) are credited and that the original publication in this journal is cited, in accordance with accepted academic practice. No use, distribution or reproduction is permitted which does not comply with these terms. 